\title{
Article
}

\section{The Mid-Atlantic Twin Registry of Virginia Commonwealth University}

\author{
Emily C. H. Lilley ${ }^{1}$, Anne-Taylor Morris ${ }^{1}$ and Judy L. Silberg ${ }^{2}$ \\ ${ }^{1}$ Mid-Atlantic Twin Registry (MATR), Virginia Commonwealth University, Richmond, VA, USA and ${ }^{2}$ Human Genetics, Virginia Commonwealth University, \\ Richmond, VA, USA
}

\begin{abstract}
The Mid-Atlantic Twin Registry (MATR) is a population-based registry of more than 60,000 twins primarily born or living in Virginia, North Carolina and South Carolina. Researchers may utilize the MATR for administration of research services, including study recruitment, data or sample (e.g., DNA) collection, archival dataset creation, as well as data collection through mailed, phone or online surveys. In addition, the MATR houses the MATR Repository, with over 1700 DNA samples primarily from whole blood available for researchers interested in DNA genotyping. For over 40 years MATR twins have participated in research studies with investigators from a range of scientific disciplines and institutions. These studies, which have resulted in numerous publications, explored diverse topics, including substance use, smoking behaviors, developmental psychopathology, bullying, children's health, cardiovascular disease, cancer, the human microbiome, epigenetics of aging, children of twins and sleep homeostasis. Researchers interested in utilizing twins are encouraged to contact the MATR to discuss potential research opportunities.
\end{abstract}

Keywords: Twin; registry; genetics; repository; recruitment

(Received 30 May 2019; accepted 2 July 2019; First Published online 14 October 2019)

\section{History and Summary}

The Mid-Atlantic Twin Registry (MATR) is a population-based registry of twins and their families that was formed through the convergence of the Virginia Twin Registry (est. circa 1975) and the North Carolina Twin Registry in 1997 and the addition of South Carolina in 1998. To date, the number of registered twins ('participants') in the MATR totals more than 60,000, with around 54,000 of these individuals representing complete pairs of same-sex and opposite-sex twins (see Tables 1 and 2). These participants have been drawn primarily from the more than 307,000 identical and fraternal twins born in these three states between 1913 and 2017, although twins from all over the United States are registered. The MATR is located administratively in the Office of Research and Innovation at Virginia Commonwealth University (VCU). The MATR facilitates twin studies for researchers from a myriad of disciplines. Researchers may utilize the MATR for administration of research services, including study recruitment, data and sample (e.g., DNA) collection, archival dataset creation, as well as data collection through mailed, phone or online surveys. Over the years, MATR twins and their family members have participated in numerous research studies (see the 'Publications and Citations' section) as well as in the MATR's data and sample collection efforts (see the 'Research Resources' section).

Author for correspondence: Emily C. H. Lilley, Email: echlilley@vcu.edu

Cite this article: Lilley ECH, Morris A-T, and Silberg JL. (2019) The Mid-Atlantic Twin Registry of Virginia Commonwealth University. Twin Research and Human Genetics 22: 753-756, https://doi.org/10.1017/thg.2019.87

\section{Methods}

\section{Twin Ascertainment}

The MATR's primary source of twin ascertainment is through birth records obtained from Virginia, North Carolina and South Carolina. In previous years, the MATR also conducted large-scale school recruitment to ascertain juvenile twin pairs. These methods are described in further detail elsewhere (Anderson et al., 2002). In the years 2014 and 2015, the MATR conducted smaller-scale ascertainment efforts through a small number of counties in Virginia. During these efforts, brochures containing information about the MATR were provided to schools either for distribution to parents of twins through 'backpack mail' or for placement in school offices. In 2010, VCU received a Clinical \& Translational Science Award (CTSA), supporting the progress of interdisciplinary research, networking and community involvement.

\section{Registration}

Prior to participating in MATR studies, twins and/or their families must first register in the MATR. Adult twins (aged 18 and older) can register individually. Juvenile twin pairs (aged 17 and younger) must be registered by their parent(s) or legal guardian(s). Registration involves providing demographic and contact information as well as answering basic zygosity questions. When contacting twins regarding registration, the MATR typically sends a postcard to the twin or twin family in advance, which serves as a simple way to introduce the MATR to them and provides a website where they can register online. Approximately 2 weeks later, the MATR sends a registration brochure to non-responders. This registration brochure contains additional information about the MATR, consent language for registration, a tear-off registration form and a postage-paid 
Table 1. Twins and multiples in sets registered in the MATR by pair sex and age group

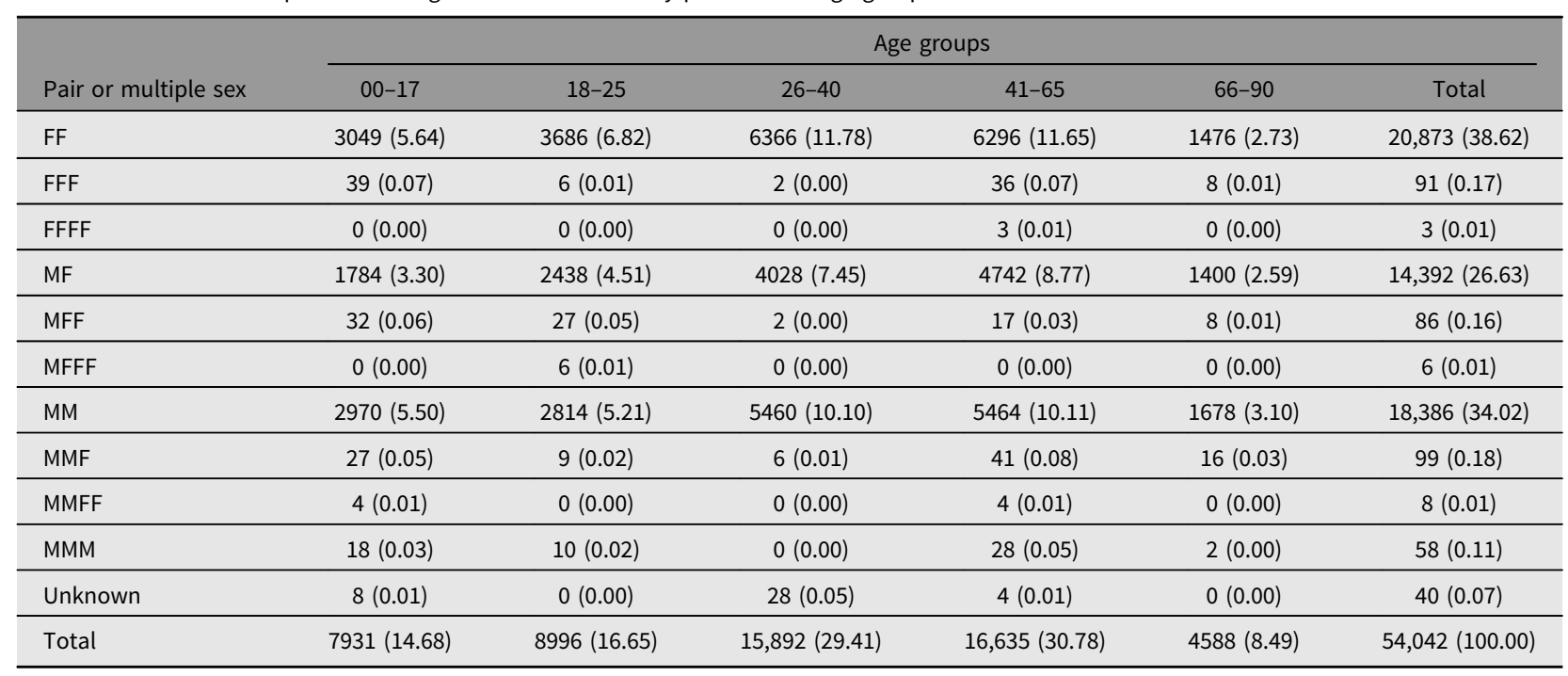

F, Female; M, Male.

Note: Each F or M represents an individual within a set of twins or higher-order multiples. Numbers given as frequency (\%) as of May 2019.

Table 2. Twins and multiples registered in the MATR by race

\begin{tabular}{lc}
\hline Race & Frequency (\%) \\
\hline Asian or Pacific Islander & $279(0.52)$ \\
\hline African American & $7076(13.09)$ \\
\hline Hispanic & $402(0.74)$ \\
\hline American Indian or Alaskan & $149(0.28)$ \\
\hline Multiracial & $1083(2.00)$ \\
\hline Other & $434(.80)$ \\
\hline Unknown & $3498(6.47)$ \\
\hline Caucasian & $41,121(76.09)$ \\
\hline Total & 54,042 \\
\hline
\end{tabular}

business-reply envelope so that they can complete registration by mail. It is a glossy, four-panel $6 \times 9$ mailer meant to be more eye-catching than university envelopes and stationery packets that were previously used. If no response is received within 2-4 weeks after sending the registration brochure, MATR research interviewers follow up via phone. During the phone call, the interviewers provide the twin or parent/legal guardian of twins with information about the MATR and invite them to register.

Some twins and their families may discover the MATR through various sources such as the internet, word of mouth or an outreach event. These twins typically register in person at an event, request a mailed packet or phone call, or sign up online through the MATR registration web survey.

\section{Study Recruitment}

Once registered in the MATR, participants are eligible to receive invitations for research studies. Participants are introduced to these studies by mail, email or phone, with the entire recruitment process commonly involving a combination of contact methods. This helps to ensure providing options that are most convenient to the participant, as well as increasing the likelihood that the preferred contact method will result in the participant encountering the study invitation materials. Typically, study invitations are initiated with an introductory letter or email, which includes a brief overview of the study. These introductory materials often also include a mechanism (mailed or online form) for the participants to provide their current contact information and to indicate their level of interest in study participation. Approximately 1 week after the initial invitation is sent, if there is no response, the MATR interviewers begin calling the twin. Generally, if the interview is not completed after 5-7 phone calls, the MATR sends a 'Trying to Reach You' letter or email to the twin, which encourages them to return the call or answer the phone and let the interviewer know whether or not they are interested in the study.

\section{Zygosity Determination}

During registration, twin zygosity is determined through a physical similarities questionnaire based on Nichols and Bilbro (1966). Many subsidiary studies that collect DNA for genotyping purposes also conduct DNA zygosity testing, and with consent, the results of the tests are returned to the MATR for record-keeping. The manner of data collection and the zygosity determination are tracked in the MATR database and are updated if more confident data are collected for the twin pair. For instance, if zygosity for a pair is initially determined by questionnaire, but then DNA testing results are obtained, this is updated as the source of zygosity rather than questionnaire determination.

\section{Tracking Twins and Twin Families}

The MATR uses a variety of public and private resources to obtain current contact information for twins and their families. Given the frequency with which the US population moves, maintaining current contact information for participants is vital to the success of the registry. Sources used by the MATR for tracking twins include address matching with the state departments of motor vehicles (in states where such access is permissible) or through matches with the National Change of Address (NCOA) database with the US Post Office. The MATR also utilizes various privately managed, 
nationwide search databases that pull information from a variety of sources such as credit header information and public records.

In addition to tracking twins through these sources, the MATR encourages twins to proactively update their contact information by phone, email or completing an online form. This allows twins to provide their current contact information in a convenient manner to ensure continued communication as well as conserve valuable department resources.

\section{Research Resources}

\section{MATR Repository}

In addition to the registry, the MATR is cultivating a repository that currently consists of blood samples from more than 1700 twins, with over 1250 of those samples representing complete pairs. Many of these samples have undergone DNA extraction and are available for researchers looking to conduct a range of genomic analysis. Twins who provided blood samples also consented for the MATR to connect the sample with their previously collected phenotypic data, such as developmental data on a number of psychiatrically relevant phenotypes. In doing so, the MATR can facilitate researcher collaboration for extensive psychiatric and genomic analyses. The VCU laboratory housing the MATR Repository is available to provide a variety of services. Interested researchers may contact the MATR Administrator or Scientific Director for access to this resource.

\section{MATR Adult Health Surveys}

The MATR collects and stores health and behavior data from adult twins in order to screen and select participants for research studies targeting specific diseases or other characteristics. These data are available for researchers interested in conducting archival data analysis.

Adult Health and Personality. The Adult Health and Personality (AHP) survey was administered from 1999 to 2002, with 3882 adult participants and over 1200 of those individuals representing complete pairs. This survey contained questions regarding height, weight, personality attributes, depression, alcohol and tobacco use, major diseases and conditions, and number of offspring.

Online MATR Adult Survey. Previously, the MATR piloted an online MATR Adult Survey (MAS) for adult twins. The MAS collects information similar to the AHP survey, as well as additional diseases, conditions, attitudes, behaviors, and information about substance use. The web-based platform provides a cost-effective and immediate method of data collection by streamlining the data directly from participant to the MATR database. Future plans for this method include using web-based applications for continuing this data collection effort with the ultimate goal of inviting all registered, adult twins to take part. In addition, the MATR hopes to deploy a similar survey to juvenile twins. These surveys will aim to collect relevant phenotypic data from MATR registrants and ascertain which registrants are willing to contribute DNA samples to the MATR Repository.

\section{Twin Communications and Outreach}

\section{Twin Matters Newsletter}

Maintaining contact with our participants is an essential component of participant engagement and longevity for the registry. The MATR produces a biannual newsletter, Twin Matters, for twins and their families, as one method of continued contact with participants.
The newsletters include twin stories, study announcements and updates, as well as articles of interest to twins. Advertisements for research studies are also included in the MATR newsletter, following (institutional review board) IRB approval.

\section{Events}

The MATR attends several events a year in efforts to raise name recognition, awareness of twin research, engagement with participants and for registration of new members. This includes twin-specific events such as regional or local Mothers of Multiples club gatherings or public events such as health fairs and expos.

\section{On the Web}

The MATR website provides information for twins and twin families as well as researchers. Twins can learn more about the MATR, twin research, current studies, and can peruse articles about common questions pertaining to twins. Researchers can find information about recent publications, MATR services and statistics about MATR participants. In addition, the MATR is developing a presence on social media by managing a Facebook page where we can share twin-related information and interact with our participants.

\section{MATR Administration and Research}

The MATR is an active human subjects protocol at VCU, which is overseen by the VCU IRB. Protecting the confidentiality and privacy of participants is of utmost importance, and the MATR employs many electronic and physical safeguards to do so, including firewalled computers, encrypted data servers and microchip (controlled-access) office entry. In addition, all MATR employees undergo training in human subjects protection and MATR Standard Operating Procedures, sign confidentiality agreements and are privy only to the least amount of information needed for them to perform their jobs.

The MATR is overseen by the Scientific Director and the Financial Director who, in combination with the MATR Administrator, steer the MATR toward advantageous initiatives and review applications from researchers who wish to access MATR twins for their study. The MATR encourages researchers from all institutions and fields of study to utilize the MATR in order to fulfill their twin-related research goals. Interested researchers may visit www.matr.vcu.edu for information on the application process and how to contact the MATR Administrator or Scientific Director.

\section{Services}

Researchers utilize the MATR for a variety of services including: (1) creating archival datasets; (2) matching data from various public and private resources; (3) conducting mailed, online or phone data collection efforts; (4) orchestrating in-home interviews; (5) coordinating large- or small-scale participant recruitment; (6) facilitating researcher networking; (7) accessing or collecting DNA or other biological samples.

The MATR provides many years of experience in overseeing study recruitment and data collection methods, as well as navigating study approvals through various IRBs.

\section{Publications and Citations}

In addition to those listed in the articles about the MATR from 2002 (Anderson et al., 2002) and 2013 (Lilley \& Silberg, 2013), the MATR 
has worked with investigators and collaborators from the VCU Departments of Human Genetics, Microbiology and Immunology, Nursing, Psychiatry, Psychology, Epidemiology, Health Behavior and Policy and the Institute for Drug and Alcohol Studies, as well as institutions such as New York University Medical Center, University of Southern California, Tulane University, Pennsylvania State and the University of Michigan. Recently published articles arising from studies that utilized MATR twins include such topics as candidate genes associated with alcohol dependence (Clark et al., 2017), bullying (Silberg et al., 2016), smoking behaviors (Do et al., 2018; Maes et al., 2018), substance use (Verhulst et al., 2014), anxiety (Hettema et al., 2015; Sawyers et al., 2019), internalizing conditions (Rappaport et al., 2017, 2018), depression (Kendler et al., 2018), anthropometric traits (Silventoinen et al., 2015), sleep homeostasis (Kuna et al., 2012), childhood sexual abuse (York et al., 2013), and methylation and healthy aging (Kim et al., 2018). Additional recent study topics include the human brain development and the impact of substance use, development of anxiety in prepubescent, adolescent and young adult twins, smoking in adolescent and young adult twins, implicit bias, genetics of aging, heritability of breast cancer, the interplay between diabetes and depression and the microbiome's impact on psoriasis and psoriatic arthritis.

In publications, MATR twins are sometimes cited under names of previous studies such as the Virginia Twin Registry, the Virginia Twin Study of Adolescent Behavioral Development (VTSABD), the Children of Twins Study (COT), the Young Adult Follow Up (YAFU), the Virginia 30,000 (VA30K) and the Virginia Adult Twin Study of Psychiatric and Substance Use Disorders (VATSPSUD).

\section{References}

Anderson, L. S., Beverly, W. T., Corey, L. A., \& Murelle, L. (2002). The Mid-Atlantic Twin Registry. Twin Research, 5, 449-455.

Clark, S. L., McClay, J. L., Adkins, D. E., Kumar, G., Aberg, K. A., Nerella S., ... van den Oord, E. J. (2017). Deep sequencing of 71 candidate genes to characterize variation associated with alcohol dependence. Alcoholism, Clinical and Experimental Research, 41, 711-718.

Do, E. K., Prom-Wormley, E. C., Eaves, L. J., Silberg, J. L., Miles, D. R., \& Maes H. H. (2018). Genetic and environmental influences on smoking behavior across adolescence and young adulthood in the Virginia Twin Study of adolescent behavioral development and the transitions to substance abuse follow-up. Twin Research and Human Genetics, 18, 43-51.

Hettema, J. M., Aggen, S. H., Kubarych, T. S., Neale, M. C., \& Kendler, K. S. (2015). Identification and validation of mixed anxiety-depression. Psychological Medicine, 45, 3075-3084.
Kendler, K. S., Gardner, C. O., Neale, M. C., Aggen, S., Heath, A., Colodro-Conde, L., ... Gillespie, N. A. (2018). Shared and specific genetic risk factors for lifetime major depression, depressive symptoms and neuroticism in three population-based twin samples. Psychological Medicine, 19, 1-9.

Kim, S., Wyckoff, J., Morris, A.T., Succop, A., Avery, A., Duncan, G. E., \& Jazwinski, S. M. (2018). DNA methylation associated with healthy aging of elderly twins. GeroScience, 40, 469-484.

Kuna, S. T., Maislin, G., Pack, F. M., Staley, B., Hachadoorian, R., Coccaro, E. F., \& Pack, A. I. (2012). Heritability of performance deficit accumulation during acute sleep deprivation in twins. Sleep, 35, 1223-1233.

Lilley, E. C., \& Silberg, J. L. (2013). The Mid-Atlantic Twin Registry, revisited. Twin Research and Human Genetics, 16, 424-428.

Maes, H. H., Morley, K., Neale, M. C., Kendler, K. S., Heath, A. C., Eaves, L. J., \& Martin, N. G. (2018). Cross-cultural comparison of genetic and cultural transmission of smoking initiation using an extended twin kinship model. Twin Research and Human Genetics, 21, 179-190.

Nichols, R. C., \& Bilbro, Jr., W. C. (1966). The diagnosis of twin zygosity. Human Heredity, 16, 265-275.

Rappaport, L. M., Carney, D. M., Brotman, M. A., Leibenluft, E., Pine, D. S., Roberson-Nay, R., \& Hettema, J. M. (2018). A population-based twin study of childhood irritability and internalizing syndromes. Journal of Clinical Child and Adolescent Psychology, 30, 1-11.

Rappaport, L. M., Sheerin, C., Savage, J. E., Hettema, J. M., \& Roberson-Nay, R. (2017). Clinical characteristics of latent classes of CO2 hypersensitivity in adolescents and young adults. Behaviour Research and Therapy, 93, 95-103.

Sawyers, C., Oliendisk, T., Brotman, M. A., Pine, D. S., Leibenluft, E., Carney, D. M., Roberson-Nay, R., \& Hettema, J. M. (2019). The genetic and environmental structure of fear and anxiety in juvenile twins. American Journal of Medical Genetics Neuropsychiatric Genetics, 180, 204-212.

Silberg, J. L., Copeland, W., Linker, J., Moore, A. A., Roberson-Nay, R., \& York, T. P. (2016). Psychiatric outcomes of bullying victimization: A study of discordant monozygotic twins. Psychological Medicine, 46, 1875-1886.

Silventoinen, K., Jelenkovic, A., Sund, R., Honda, C., Aaltonen, S., Yokoyama, Y., ... Kaprio, J. (2015). The CODATwins Project: The cohort description of collaborative project of development of anthropometrical measures in twins to study macro-environmental variation in genetic and environmental effects on anthropometric traits. Twin Research and Human Genetics, 18, 348-360.

Verhulst, B., Eaves, L. J., \& Neale, M. C. (2014). Moderating the covariance between family member's substance use behavior. Behavior Genetics, 44, 337-346.

York, T. P., Brumelle, J., Juusola, J., Kendler, K. S., Eaves, L. J., Amstadter, A. B., ... Jackson-Cook, C. (2013). Increased frequency of micronuclei in adults with a history of childhood sexual abuse: A discordant monozygotic twin study. PLoS One, 8, e55337. 